\title{
APLICAÇÃO DA TERMOGRAFIA INFRAVERMELHA NA IDENTIFICAÇÃO DE MANIFESTAÇÕES PATOLÓGICAS NAS CONSTRUÇÕES
}

\author{
DANTAS NETO, JOÃO ANTONIO \\ Graduando em Engenharia Civil \\ UFERSA \\ Rio Grande do Norte; Brasil \\ joaodantasufersa@gmail.com
}

\author{
BESSA, CARLOS VINÍCIUS DAMACENO \\ Docente em Engenharia Civil \\ UFERSA \\ Rio Grande do Norte; Brasil \\ vinibessasp@hotmail.com
}

\author{
SILVA, ILG PATRICK DANTAS \\ Engenheiro Civil \\ UFERSA \\ Rio Grande do Norte; Brasil \\ ilg@grupobrisanet.com.br
}

\author{
MAIA, GEOVANNA PAULINA DANTAS \\ Graduanda em Engenharia Mecânica \\ UFERSA \\ Rio Grande do Norte; Brasil \\ maia.geovanna@gmail.com
}

\author{
DANTAS, LUCAS RAMOS \\ Docente em Engenharia Civil \\ UFERSA \\ Rio Grande do Norte; Brasil \\ lucas.dantas@ufersa.edu.br
}

\section{RESUMO}

A termografia infravermelha é uma ferramenta de inspeção não intrusiva e não destrutiva que permite a geração de imagens termográficas mediante a emissividade dos materiais. A aplicação desse método na construção civil torna-se cada vez mais promissor, tendo em vista a sua eficiência na identificação das mais diversas manifestações patológicas. Diante do exposto, objetiva-se apresentar uma revisão bibliográfica dos estudos que empregaram a termografia infravermelha na análise de problemas patológicos presentes na construção civil. Além disso, será descrito as metodologias utilizadas para o aperfeiçoamento dessas análises, as influências e limitações desse processo que podem interferir na obtenção dos dados. Os resultados da pesquisa mostram que o ensaio termográfico possui uma grande aplicabilidade na identificação de manifestações patológicas, além de que proporciona o diagnóstico precoce e o monitoramento em tempo real, reduzindo tempo e custos destas atividades. No entanto, devem ser considerados alguns fatores para garantir resultados confiáveis, como as condições ambientais em que o material analisado está exposto. Palavras-chave: Avaliação não destrutiva; Revisão Bibliográfica; Construção Civil.

\section{ABSTRACT}

The infrared thermographic is a non-intrusive, non-destructive inspection tool that allows the generation of thermographic images using an emissivity of materials. The application of this method in civil construction is becoming increasingly promising, given its efficiency in identifying the most diverse pathological manifestations. Therefore, this paper aims to present a literature review of the studies that used infrared thermography in the analysis of pathological problems present in construction. In addition, we will describe the methodologies used to improve these analyzes, the influences and limitations of this process that may interfere in the obtaining of data. The research results show that the thermographic test has a great applicability in identifying pathological manifestations, besides providing early diagnosis and real time monitoring, reducing time and costs of these activities. However, some factors must be considered to ensure reliable results, such as the environmental conditions under which the material analyzed is exposed.

Keywords: Non-destructive inspection; Literature Review; Civil Construction.

\section{INTRODUÇÃO}

Toda matéria que possui uma temperatura acima do zero absoluto $\left(-273,15^{\circ} \mathrm{C}\right)$ emite energia térmica através da radiação infravermelha. Contida na região invisível do espectro eletromagnético, a radiação infravermelha é caracterizada por ter comprimentos de onda entre 700 nanômetros a 1 milímetro (VERMA; GOH, 2019). Essa radiação 
não pode ser observada a olho nu, apenas quando a temperatura do material excede 500 graus Celsius. Logo, para converter essa energia infravermelha em uma imagem visual bidimensional, utiliza-se câmeras termográficas que fornecem o mapeamento da distribuição de temperaturas na superfície do objeto fotografado (GHORI, 2010). Desse modo, pode-se dizer que a termografia infravermelha é uma representação gráfica do calor.

Segundo Gade e Moeslund (2013), o primeiro dispositivo infravermelho foi usado para fins militares, buscando-se uma melhor visão noturna. No entanto, após o avanço dessa tecnologia, foi possível perceber que as câmeras termográficas, além de proporcionar uma melhor visão, são capazes de revelar e calcular facilmente diferenças irregulares de temperatura nas superfícies. Desse modo, a termografia ganhou espaço nas mais diversas áreas, sendo utilizada para a realização de análises em animais, agricultura, edifícios, aplicações industriais e militares, bem como para a detecção, rastreamento e reconhecimento de seres humanos. Na construção civil, observa-se que desde o final do século XX vêmse estudando aplicações e buscando a normatização da utilização dessa ferramenta (INTERNATIONAL ORGANIZATION FOR STANDARDIZATION, 1983; CHEW, 1998).

Ao longo dos anos, diversos pesquisadores investigaram a aplicabilidade da termografia infravermelha nos estudos de inspeção e avaliação de estruturas. Pesquisas mostraram a utilização dessa técnica para detectar trincas em vigas subterrâneas, corrosão em concreto armado, umidade no revestimento cerâmico de fachadas, entre outras manifestações patológicas (AGGELIS et al., 2010; KOBAYASHI e BANTHIA, 2011; EDIS et al., 2014). Um dos principais motivos que torna atrativo a utilização da termografia é que essa ferramenta proporciona a realização de análises não destrutivas e, dessa forma, não interfere na estabilidade da estrutura durante sua execução. No entanto, fatores como propriedades do material, condições ambientais e posicionamento da câmera podem interferir nos resultados obtidos.

Tendo em vista o avanço da termografia infravermelha nas últimas décadas e os benefícios que esta técnica pode proporcionar, tem-se por objetivo apresentar uma revisão bibliográfica de publicações científicas, em livros e artigos científicos de estudos aplicados em diversos países que empregaram os ensaios termográficos para identificação dos problemas patológicos presentes na construção civil. Além disso, serão descritas as metodologias utilizadas para o aperfeiçoamento destas análises, bem como as influências e limitações que podem interferir na obtenção dos dados deste processo.

\section{A TERMOGRAFIA INFRAVERMELHA}

As imagens termográficas podem ser representadas em tons de cinza, exibindo regiões quentes na cor branca e indicando as temperaturas mais baixas através de uma escala de tons de cinza até a cor preta, onde é registrado o local com a menor temperatura. Para uma melhor interpretação dos dados, são comumente utilizadas as câmeras termográficas que apresentam paletas de cores, onde branco, vermelho e amarelo simbolizam níveis mais altos de energia, enquanto que violeta, azul e preto representam os níveis mais baixos (GHORI, 2010). No entanto, essa representação escalar das cores não é padrão, tendo em vista que há diversos tipos de paletas utilizadas nas câmeras termográficas, indicadas para propósitos diferentes ou para condições específicas.

Os ensaios termográficos podem ser classificados por passivos ou ativos. No passivo, segundo Rudawska (2019), o objeto fotografado emite raios infravermelhos naturalmente e de modo suficiente para a realização da análise. Enquanto que para o ensaio ativo é utilizada outra fonte de estimulação térmica para aquecer ou resfriar o objeto, visto que os defeitos do material são de temperatura uniforme e, portanto, não emitem sinais que caracterizam essas irregularidades. Logo, neste caso, para que a superfície mostre um campo dinâmico de temperatura, é requerido uma estimulação térmica adicional. Conforme Halabe (2013), durante esse estímulo é possível perceber que a taxa de condução térmica através de um defeito geralmente é diferente da taxa de condução através de material livre de defeitos. Nas manifestações patológicas como trincas e fissuras, em que há um espaçamento que pode ser preenchido por ar, esse fluido age como um isolante térmico, causando acúmulo de calor na região superficial acima do defeito.

No entanto, é importante enfatizar que a energia registrada pela câmera infravermelha não depende apenas da temperatura do objeto, mas também da emissividade. Segundo Li (2016), a emissividade térmica de um material determina quanto calor pode ser emitido por unidade de área a uma determinada temperatura, ou seja, quão rapidamente uma superfície emite calor. Conforme Cerdeira et al., (2011), os materiais de construção mais utilizados, exceto os metais, possuem valores de emissividade altos, sendo em sua maioria superiores a 0,8. Desse modo, o uso da termografia infravermelha torna-se relevante na construção civil, principalmente quando se deseja identificar elementos estruturais, como pode ser visto na Figura 1. 

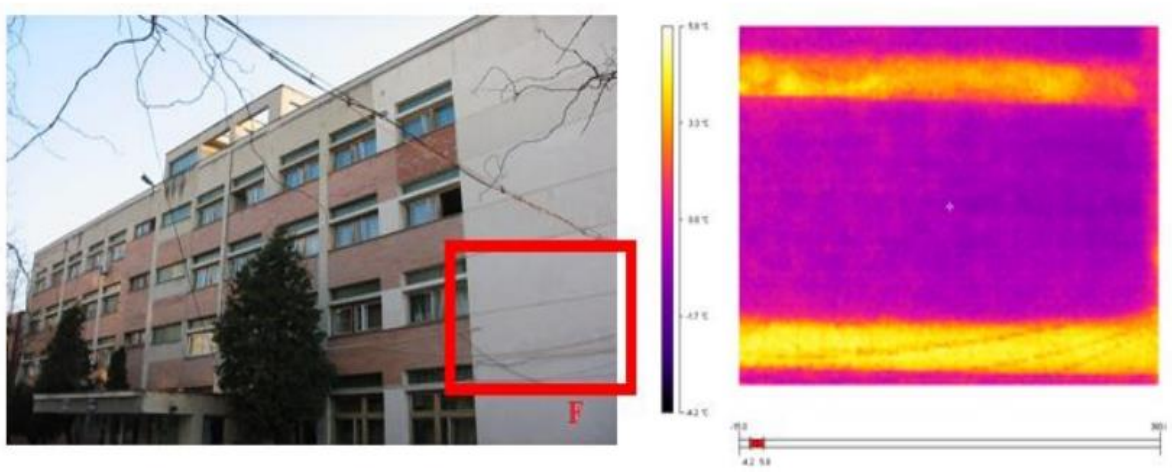

Figura 1: Termografia Infravermelha Aplicada em Estrutura de Concreto Armado.

Fonte: Budescu (2016)

Na Figura 1, observa-se as perdas térmicas próximas às vigas de concreto, em decorrência da baixa emissividade do aço comparado com a emissividade do concreto. Esse fator faz com que o aço apresente maiores temperaturas na escala do termograma, enquanto que o concreto, representado pelas cores escuras, apresente menores temperaturas. Desse modo, pode-se afirmar que, se um material possui alta emissividade, consequentemente, apresenta menores níveis de temperatura, ou vice-versa. Essa afirmação é comprovada pela lei de Stefan-Boltzmann,

$$
\mathrm{E}=\varepsilon \times \sigma \times \mathrm{T}^{4}
$$

Onde:

E corresponde a energia radiante emitida por uma superfície $\left(\mathrm{W} / \mathrm{m}^{2}\right)$

$\varepsilon$ equivale a emissividade do material

$\sigma$ é a constante de Stefan-Boltzmann $\left(5,6697 \times 10-8 \mathrm{~W} / \mathrm{m}^{2} \mathrm{~K}^{4}\right)$

$\mathrm{T}$ corresponde a temperatura da superfície (K)

Baseada nos princípios desta lei, a câmera termográfica mede a emissão de energia radiante e, através da utilização da constante de Stefan-Boltzmann e do valor tabelado da emissividade do material analisado, calcula e exibe o valor da temperatura na imagem termográfica (JANKŮ et al., 2019). Tendo em vista que através das variações de temperatura é possível realizar diagnósticos satisfatórios, diversos pesquisadores estudaram a aplicabilidade dessa ferramenta para identificar manifestações patológicas na construção civil. No tópico seguinte será descrito alguns desses problemas e como a termografia pode auxiliar no diagnóstico precoce.

\section{TERMOGRAFIA INFRAVERMELHA NA IDENTIFICAÇÃO DE MANIFESTAÇÕES PATOLÓGICAS}

As técnicas destrutivas para identificar manifestações patológicas pode ocasionar danos a estrutura. Por esse motivo, para o diagnóstico do estado de conservação das edificações do patrimônio histórico, Dorrego et al. (2003) aconselharam o uso de técnicas de avaliação com métodos não destrutivos, como termografia infravermelha. Uma década depois, Paoletti et al. (2013) apresentaram os resultados da aplicação desse método para diagnosticar a igreja de Santa Maria ad Cryptas, uma edificação da Idade Média com arquitetura românica, como pode ser visto na Figura 2. 

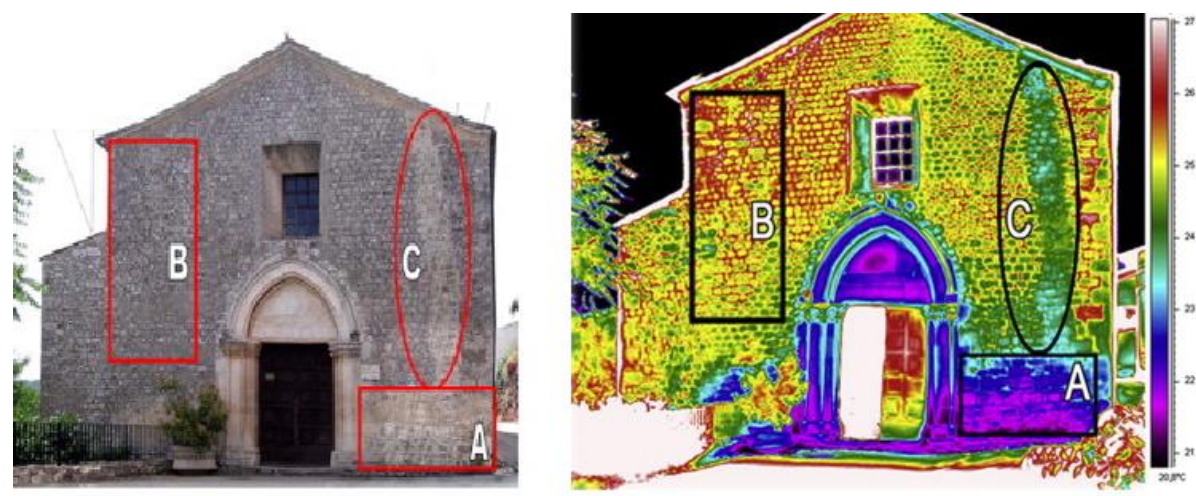

Figura 2: Análise termográfica na igreja de Santa Maria ad Cryptas.

Fonte: Paoletti et al. (2013)

Na região A, foi identificado a presença de ataque biológico em razão da umidade provinda do solo. Na região B há falta de argamassa provavelmente causada por uma série de tensões mecânicas devido a razões térmicas, produzindo a instabilidade do lado esquerdo da fachada. A heterogeneidade da área $\mathrm{C}$ testemunha o uso de diferentes tipos de argamassa, uma argamassa mais hidrófila que a original, usada em restaurações anteriores da igreja (PAOLETTI et al., 2013).

Outra finalidade para a aplicação dessa tecnologia em fachadas é a identificação de problemas patológicos em revestimentos. É visto que, nas fachadas é comum a formação de bolsas de ar entre o revestimento e a base. Atuando como barreiras térmicas, essas bolsas dificultam a transferência de energia para o interior, absorvendo a radiação solar e aumentando a temperatura local, podendo desse modo ser identificada por um termograma antes mesmo que os sinais visíveis ocorram, como pode ser observado na Figura 3 (RAPOSO, 2017).
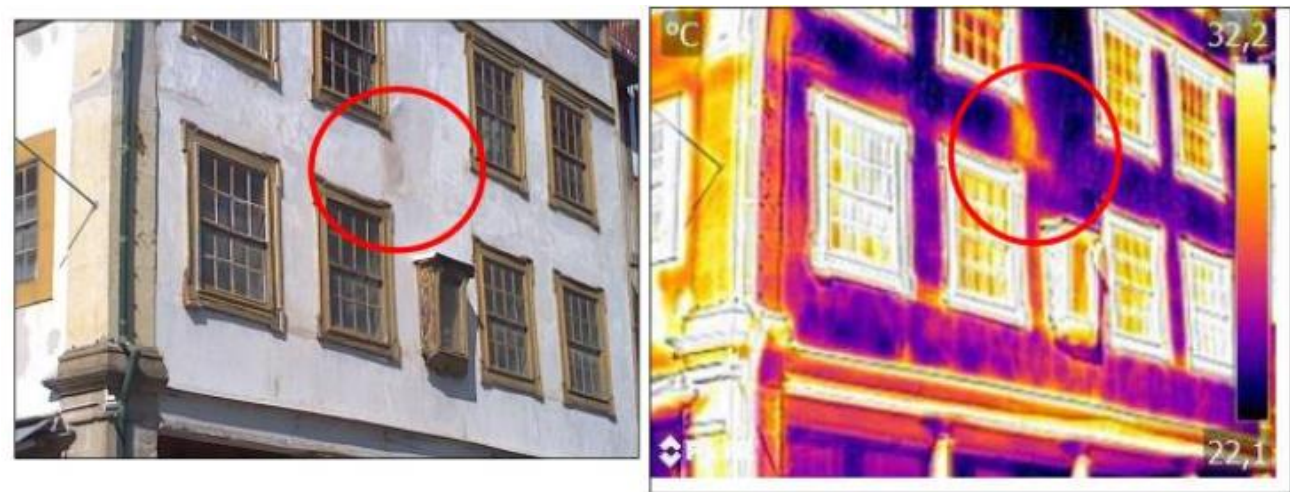

Figura 3: Análise de Manifestações Patológica em Revestimento de Fachada. Fonte: Raposo (2017)

Balaras e Argiriou (2002) indicaram a utilização de câmeras termográficas para identificar patologias em áreas de telhados e apresentaram a Figura 4, onde é possível observar um termograma em tons de cinza que indica irregularidades em uma cobertura. 


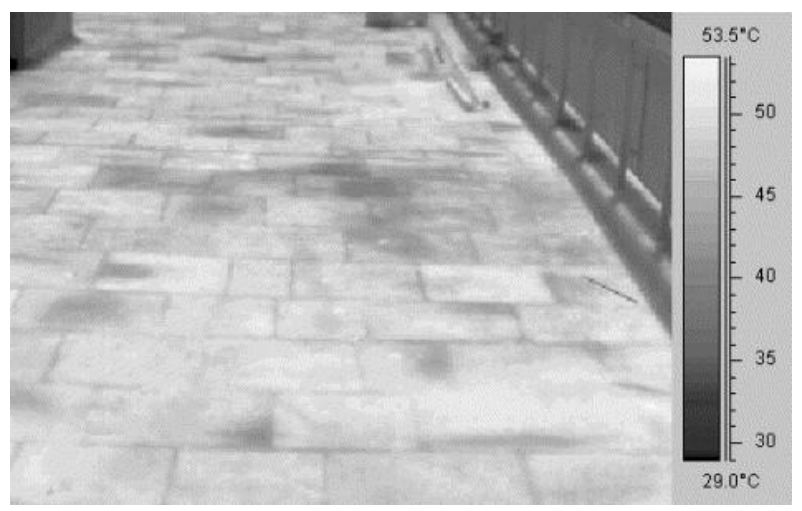

Figura 4: Análise Termográfica em Cobertura. Fonte: Balaras e Argiriou (2002)

Segundo Balaras e Argiriou (2002), na Figura 4, as regiões que contêm umidade são identificadas como áreas com temperaturas mais elevadas. De acordo com Meola e Carlomagno (2004), isso acontece em razão de que a água tem uma capacidade calorífica maior que o restante do material, desse modo ela retém o calor por um longo período de tempo e as áreas úmidas podem ser facilmente detectadas à noite após o resfriamento das áreas secas do telhado. Para analisar regiões de difícil acesso, como telhados de grandes estruturas, Ortiz-sanz et al. (2019) recomendaram a realização de registro termográficos com o auxílio de veículos aéreos não tripulados.

Segundo Raposo (2017), a termografia infravermelha proporciona grandes vantagens na análise de infiltrações decorrentes de falhas nas tubulações, tendo em vista que através dessa técnica é possível identificar anomalias e infiltrações de forma rápida e precisa. Assim, por ser um ensaio não destrutivo, a termografia acelera o processo de reabilitação da rede de abastecimento de água e, consequentemente, proporcionando uma economia significativa. A Figura 5 demonstra o quanto é nítida a identificação de tubulações em imagens termográficas.

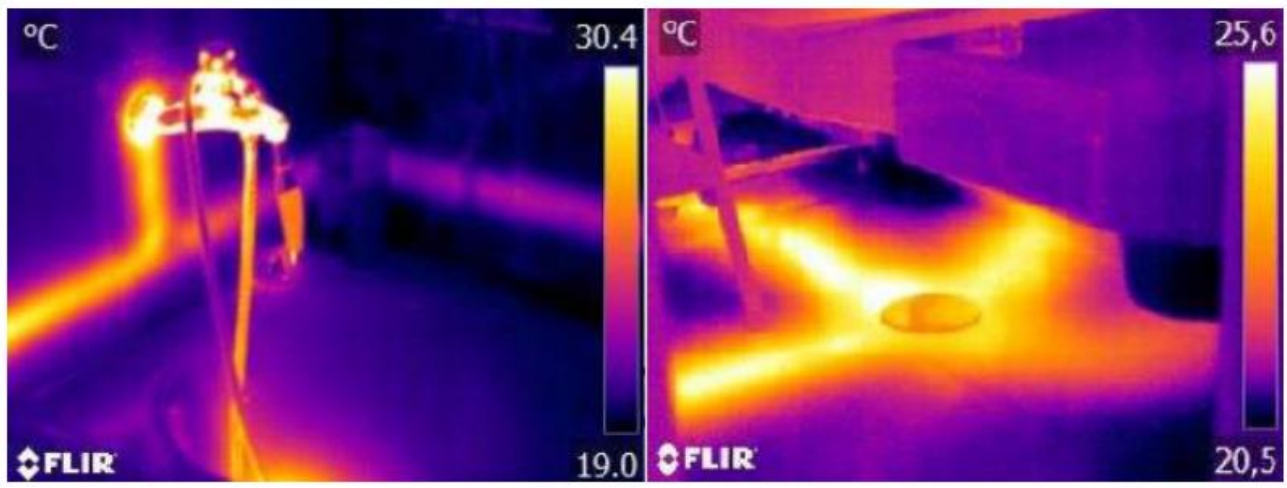

Figura 5: Exemplos de Análises em Instalações Hidrossanitárias.

Raposo (2017)

Dadas as diversas aplicabilidades da termografia infravermelha na análise de manifestações patológicas, é válido ressaltar que o uso deste tipo de ensaio requer uma avaliação criteriosa no processo de obtenção de resultados. Tendo em vista que elementos expostos as ações ambientais, como fachadas, sofrem efeitos como a variação do vento, que, por sua vez, pode reduzir a temperatura da superfície analisada. Outro exemplo de possível interferência é o caso de estruturas expostas a variação da radiação solar ao longo do dia ou até mesmo ao resfriamento promovido pela ocorrência de chuvas. Diante disto, de acordo com Meola (2007) deve-se buscar minimizar a ocorrência de tais interferências, através da realização de capturas de imagens durante a noite ou durante dias nublados, reduzindo a perda de calor por convecção.

Outra limitação do método é a baixa disseminação de sua aplicação no país, desta forma, tem-se um número reduzido de profissionais especializados em sua execução, algo que pode proporcionar uma certa resistência no processo de popularização do método no mercado da construção civil brasileiro. 


\section{CONCLUSÃO}

De acordo com os resultados, verifica-se que o ensaio é eficiente para deteç̧ão de danos estruturais, possibilitando medidas de restauração ou reforço, antes do colapso das estruturas. Além disso, pode-se dizer que é viável na investigação de infiltrações, que podem ocasionar o surgimento de manifestações patológicas como formação de mancha ou bolor, eflorescência e desagregação de revestimentos e forros. Outra possibilidade de aplicação, possível de averiguação em estudos futuros, é a análise comparativa de diferentes materiais, relacionando os resultados obtidos com a qualidade que estes materiais possuem, como por exemplo, o estudo de argamassas aplicadas em diferentes pontos de uma estrutura.

Dentre as principais vantagens desta ferramenta de inspeção, pode-se destacar a não necessidade de extração de um testemunho do elemento analisado, ou seja, trata-se de um ensaio não destrutivo. Com isso, tem-se a possibilidade da obtenção de resultados em elementos de difícil acesso, como é o caso de telhados, fachadas e vigas subterrâneas. Além disso, tal condição não causa danos a estabilidade da estrutura, algo que viabiliza a aplicação de diversos ensaios em uma mesma região e a avaliação de estruturas vulneráveis a grandes alterações, como patrimônios históricos.

No que se diz respeito as principais desvantagens deste mecanismo de análise, sobressai-se a falta de popularização de sua aplicação, algo que é refletido na ausência de sua normatização e promove o surgimento de deficiências como o fato da representação escalar das cores exibidas pelo ensaio, não ser padrão. Além disto, destaca-se a existência de fatores ambientais que podem alterar os resultados obtidos e devem ser considerados no momento de execução, como a variação do vento, a variação de da radiação solar ao longo do dia e o resfriamento ocorrido pela ocorrência de chuvas.

Outra sugestão que pode ser adotada em estudos futuros é a avaliação do conforto térmico em edificações por meio da aplicação da termografia infravermelha.

\section{REFERÊNCIAS}

AGGELIS, D. G. et al. Combined use of thermography and ultrasound for the characterization of subsurface cracks in concrete. Construction and Building Materials, v. 24, n. 10, p.1888-1897, out. 2010.

AVDELIDIS, N. P.; MOROPOULOU, A. Emissivity considerations in building thermography. Energy and Buildings, v. 35, n. 7, p. 663-667, ago. 2003.

BALARAS, C. Argitiou; ARGIRIOU, A. A. Infrared thermography for building diagnostics. Energy and buildings, v. 34, n. 2, p. 171-183, 2002.

BUDESCU, M. Using thermography and artificial neural networks in civil engineering. v. 62, n. 66, 2016

CHEW, M. Y. L. Assessing building façades using infra-red thermography. Structural Survey, 1998.

CERDEIRA, F. et al. Applicability of infrared thermography to the study of the behaviour of stone panels as building envelopes. Energy and Buildings, v. 43, n. 8, p. 1845-1851, 2011.

DORREGO, J.; LUXÁN, M. P.; DORREGO, F. Damage detection and localization of reinforcement elements in historic buiildings with infrared thermography. In: International Conference on Advances in Concrete and Structures. RILEM Publications SARL, 2003. p. 567-574.

EDIS, Ecem; FLORES-COLEN, Inês; DE BRITO, Jorge. Passive thermographic detection of moisture problems in façades with adhered ceramic cladding. Construction and Building Materials, v. 51, p. 187-197, 2014.

GADE, Rikke; MOESLUND, Thomas B. Thermal cameras and applications: a survey. Machine vision and applications, v. 25, n. 1, p. 245-262, 2014.

GHORI, Salahuddin. Infrared (IR) Thermography for Condition Monitoring at Q-Chem. Proceedings of the 2nd Annual Gas Processing Symposium, p.209-219, 2010.

HALABE, U. B. Non-destructive evaluation (NDE) of composites: techniques for civil structures. In: Non-Destructive Evaluation (NDE) of Polymer Matrix Composites. Woodhead Publishing, 2013. p. 483-517e. 
INTERNATIONAL ORGANIZATION FOR STANDARDIZATION. ISO 6781: Thermal insulation - Qualitative detection of thermal irregularities in building envelopes - Infrared methods. Switzerland, 1983.

JANKŮ, Michal et al. Comparison of infrared thermography, ground-penetrating radar and ultrasonic pulse echo for detecting delaminations in concrete bridges. Construction and Building Materials, v. 225, p.1098-1111, nov. 2019.

KOBAYASHI, K.; BANTHIA, N. Corrosion detection in reinforced concrete using induction heating and infrared thermography. Journal of Civil Structural Health Monitoring, v. 1, n. 1-2, p. 25-35, 2011.

LI, Hui. Literature Review on Cool Pavement Research. Pavement Materials for Heat Island Mitigation, p.15-42, 2016.

MEOLA, Carosena; CARLOMAGNO, Giovanni M. Recent advances in the use of infrared thermography. Measurement Science and Technology, v. 15, n. 9, p.27-58, 24 jul. 2004.

ORTIZ-SANZ, Juan et al. IR Thermography from UAVs to Monitor Thermal Anomalies in the Envelopes of Traditional Wine Cellars: Field Test. Remote Sensing, v. 11, n. 12, p.1-18, 14 jun. 2019.

PAOLETTI, Domenica et al. Preventive thermographic diagnosis of historical buildings for consolidation. Journal of Cultural Heritage, v. 14, n. 2, p.116-121, mar. 2013.

RAPOSO, Nuno Miguel Rocha. Diagnóstico de patologias na construção apoiada na análise termográfica. Lisboa: Instituto Superior de Engenharia de Lisboa, 2017. Dissertação de mestrado.

RUDAWSKA, Anna. Bonding technology. Surface Treatment in Bonding Technology, p.7-46, 2019.

VERMA, Deepak; GOH, Kheng Lim. Natural fiber-reinforced polymer composites. Biomass, Biopolymer-based Materials, and Bioenergy, p.51-73, 2019. 